\title{
Commentary
}

\section{Cervical cancer elimination in the era of COVID-19: the potential role of Artificial Intelligence (AI)-guided digital colposcope cloud platform}

\author{
Maria Jose Gonzalez Mendez ${ }^{1, \dagger}$, Peng Xue ${ }^{2, *, \dagger}$, Youlin Qiao ${ }^{1,2, *}$ \\ ${ }^{1}$ School of Public Health, Dalian Medical University, 116044 Dalian, Liaoning, China \\ ${ }^{2}$ School of Population Medicine and Public Health, Chinese Academy of Medical Sciences and Peking Union Medical College, 100730 Beijing, China \\ *Correspondence: xuepeng_pumc@foxmail.com (Peng Xue); qiaoy@cicams.ac.cn (Youlin Qiao) \\ $\dagger$ These authors contributed equally. \\ Academic Editor: Enrique Hernandez \\ Submitted: 19 November 2021 Revised: 12 December 2021 Accepted: 14 December 2021 Published: 15 February 2022
}

During the last years, the world has been stunned by an emerging health outbreak emergency, the COVID-19 disease, which has spread worldwide. The COVID-19 pandemic has affected chronic diseases such as cervical cancer due to the postponement of health services and the interruption of several cervical cancer interventions. In 2020, the World Health Organization officially launched the cervical cancer elimination strategy, and efforts must be made irrespective of the pandemic. Artificial Intelligence (AI)guided digital colposcope cloud platform could be especially relevant during the COVID-19 era, and together with other innovative screening methods, could accelerate cervical cancer elimination. The COVID-19 pandemic period could be an opportunity to improve cervical cancer screening services and get closer to the target of eliminating cervical cancer by 2030 .

According to the World Health Organization (WHO), cervical cancer (CC) is the fourth most common cancer in women worldwide. Around 341,800 women died from this disease in 2020 [1], and the cases continue to grow. However, CC is a preventable and curable disease if detected early. In May 2018, the Director-General of WHO, Dr. Tedros Adhanom, announced a global call to eliminate CC. He mentioned we can eliminate $\mathrm{CC}$ as a Public Health problem and make it a disease of the past through cost-effectiveness, evidence-based interventions, including human papillomavirus (HPV) vaccination of girls, screening and treatment of precancerous lesions, and improving access to diagnosis and treatment of invasive cancers [2]. In November 2020, WHO officially launched the CC elimination strategy [3].

However, the coronavirus SARS-CoV-2 (COVID-19) pandemic started in 2019 led to a halt in health services worldwide [4]. Patients with chronic diseases, such as $\mathrm{CC}$, have been affected indirectly by the pandemic due to the postponement of services and the interruption of several CC-related interventions. In many countries, the CC screening and immunization programs were delayed due to the pandemic. Data suggest that COVID-19 has created challenges for initiatives to recruit under-screened women, as cervical screening requires in-person attendance at the clinic [5]. In low-resource settings, the screening personnel was re-allocated, or the staff members were sent home because of various levels of lockdown. As a result, cancer screening temporarily stopped being a priority [6].

The COVID-19 pandemic has highlighted the necessity of flexible and highly efficient health systems since the world could eventually be affected by another emergency that forces CC services to stop. Several adjustments have been observed worldwide, such as transitioning to web-based consultations or telemedicine [7], restricting personnel to an absolute minimum, and the use of home-based self-collection (supported by telehealth), such as home-based screening (HPV self-sampling). HPV selfsampling has proven to be equivalent to clinician sampling and quite practical and teachable by simple graphics or animated video presentations, as demonstrated in studies from Nigeria [8]. The new urgency of the COVID-19 pandemic support self-sampled HPV testing as the primary cervical screening method, which could decrease the number of women attending the hospital clinic [9].

The COVID-19 pandemic era may be the best period to implement technologies that make the screening system more efficient and less dependent on the training of health professionals. In this regard, artificial intelligence (AI) methods have demonstrated adequate capacity in prediction and diagnoses for malignancies, such as breast cancer, colorectal cancer, and gastrointestinal cancer [10]. Thus, in CC screening, colposcopy is another procedure that must advance and become more efficient to avoid the bottleneck that limits screening performance. In China, an AIguided digital colposcopy cloud platform that enables clinicians to predict biopsies was developed and validated by Chinese scientists through a project that started in 2018 [11]. This platform based on uploading images and information into a cloud, can provide real-time diagnostic results. It focuses on imaging characteristics and integrates correlated factors, such as age, into the diagnosis. In addition, it combines cytology and HPV test results for performing an intelligent analysis. The platform was recently validated in China through a retrospective study performed in the Shenzhen Maternity and Child Healthcare Hospital, and 


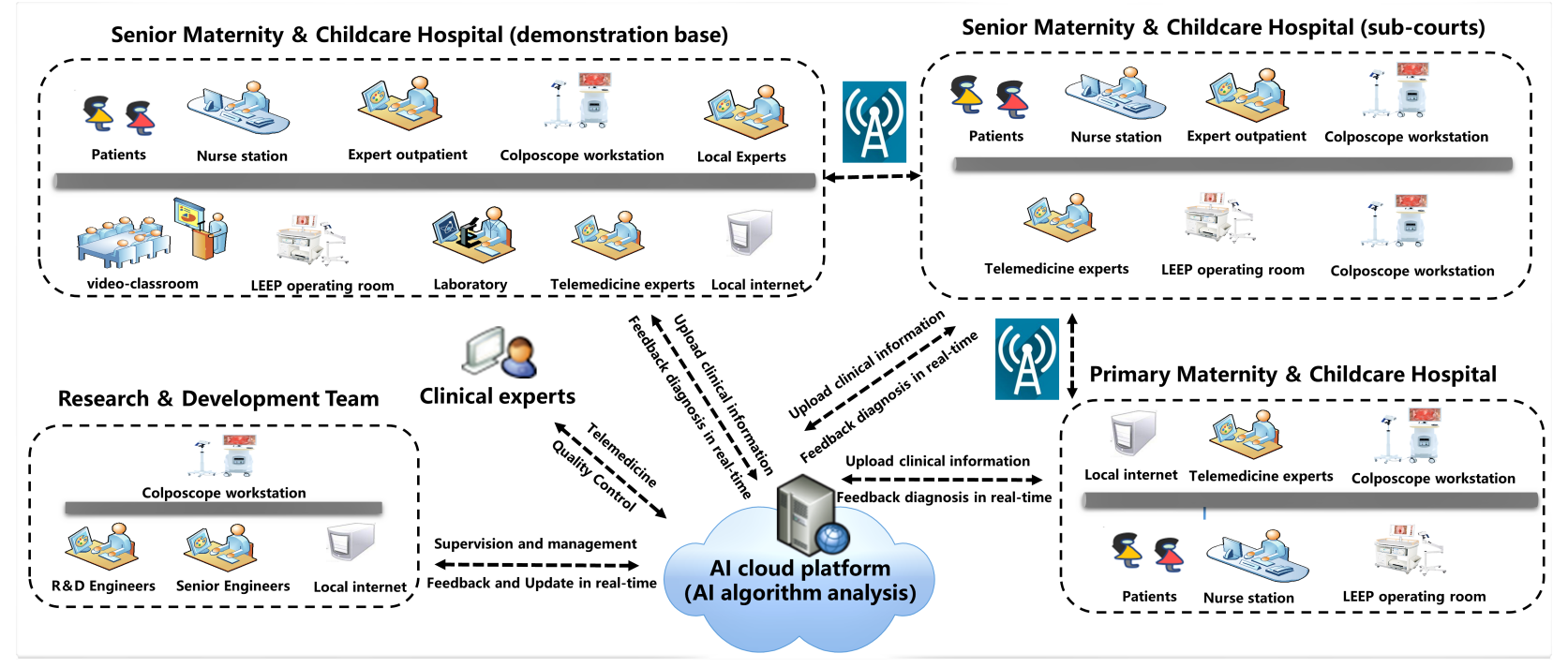

Fig. 1. Application scenario of the AI-guided digital colposcope cloud platform in Ordos City, Inner Mongolia, China.

it was published in $2020[11,12]$. More than 100,000 colposcopic images and non-image information from 19,435 patients were analyzed and compared with the pathological diagnosis (gold standard). The previously mentioned study demonstrated $82.2 \%$ agreement between the AI platform and the pathological results and was higher than the original colposcopic interpretation by colposcopists $(65.9 \%)$. Moreover, the AI platform had an excellent agreement with pathology results when distinguishing between nor$\mathrm{mal} /$ benign (95.5\%) and cancer (100\%).

Integrating AI technologies into screening services is the cornerstone of the change needed to move faster toward eliminating CC. AI trains and programs computers to learn patterns from data based on mathematical rules and statistical assumptions. The learned program or classifier, an algorithm that sorts data into categories, is then used to predict or identify a pattern. AI technique principles have been comprehensively analyzed and reported in CC screening and cancer diagnosis by the Chinese Academy of Medical Sciences research team led by Dr. Youlin Qiao $[13,14]$. They adopted an interpretable graph convolutional network framework with node and edge features (E-GCN) to fuse the extracted lesion features and yield the patient-wise classification result.

Due to its relevant role in CC, the AI-guided digital colposcope cloud platform might help perform colposcopy exams more efficiently, especially during the COVID-19 pandemic. The AI-based platform shows a sequence of the best images to the physician, which are immediately uploaded to the cloud. Then, the cloud sends feedback to get information about the best biopsy sites. Experienced colposcopists are not required, and more colposcopy units can perform this exam, helping to reduce the bottleneck challenge in colposcopy examination, which becomes more critical in hospitals and clinics when social distancing is required. It is essential to mention that the AI colposcopy cloud platform can be used as an examination tool, and it can also assist in training new colposcopists online [15]. Moreover, the AI-guided digital colposcope cloud platform helps health professionals to improve the accuracy of CC diagnosis, but it does not replace the role of the physician in the process, and the diagnosis is still made and communicated by a human, not a machine.

Proof that the integration of high technologies can help improve screening services and achieve the elimination of $\mathrm{CC}$, is the case of Inner Mongolia that has become one of the "first mover" regions in eliminating $\mathrm{CC}$ in China by promoting prevention through free HPV vaccination and CC screening [16]. In 2019, a pilot project was implemented in the city of Ordos in Inner Mongolia. The project utilized the AI-guided digital colposcopy cloud platform in senior and primary Maternity and Childcare Hospitals of several counties. Senior and primary hospitals were connected through a cloud that received the information of the colposcopy exams. All the information accumulated during several exams was stored to later send feedback to the colposcope workstation about the biopsy sites, making the examination process more efficient (Fig. 1). The case of Inner Mongolia is not only a milestone for CC in China but also a confirmation that elimination is possible regardless of difficulties if health technologies and services are adequate.

During this period, when CC services and programs have been affected, it is necessary to adapt quickly to the world's new context. The AI-guided digital colposcope cloud platform could help reduce the number of professionals and patients at the healthcare units, quickly train new colposcopists online, improve the quality of cancer diagnoses, and help low-middle-income countries (LMICs) and remote areas in improving diagnosis efficiency. The COVID-19 pandemic is bringing together experts to evaluate new strategies to support cancer control worldwide. 
This period is an opportunity to improve screening programs and accelerate $\mathrm{CC}$ elimination by incorporating new technologies and enhancing screening methods. The AI-guided digital colposcope platform combined with other innovative techniques, such as the self-sampling HPV DNA could be an efficient strategy to improve $\mathrm{CC}$ services and achieve CC elimination by 2030 .

\section{Author contributions}

MJGM, PX, and YQ designed the manuscript. MJGM and PX wrote the final article. All authors contributed to editorial changes in the manuscript. All authors read and approved the final manuscript.

\section{Ethics approval and consent to participate}

Not applicable.

\section{Acknowledgment}

We would like to express our gratitude to Didier Sama Salah who helped us editing this manuscript, and to all the peer reviewers for their opinions and suggestions.

\section{Funding}

This research was funded by CAMS Innovation Fund for Medical Sciences (Grant \#: CAMS 2021-I2M-1-004).

\section{Conflict of interest}

The authors declare no conflict of interest.

\section{References}

[1] Sung H, Ferlay J, Siegel RL, Laversanne M, Soerjomataram I, Jemal A, et al. Global Cancer Statistics 2020: GLOBOCAN Estimates of Incidence and Mortality Worldwide for 36 Cancers in 185 Countries. CA - A Cancer Journal for Clinicians. 2021; 71 : 209-249.

[2] World Health Assembly adopts global strategy to accelerate cervical cancer elimination. World Health Organization. Year. Available at: https://www.who.int/news/item/19-08-2020-wor ld-health-assembly-adopts-global-strategy-to-accelerate-cervi cal-cancer-elimination (Accessed: 19 August 2020).

[3] Das M. WHO launches strategy to accelerate elimination of cervical cancer. The Lancet Oncology. 2021; 22: 20-21.

[4] Del Pilar Estevez-Diz M, Bonadio RC, Miranda VC, Carvalho JP. Management of cervical cancer patients during the COVID-
19 pandemic: a challenge for developing countries. Ecancermedicalscience. 2020; 14: 1060.

[5] Feletto E, Grogan P, Nickson C, Smith M, Canfell K. How has COVID-19 impacted cancer screening? Adaptation of services and the future outlook in Australia. Public Health Research and Practice. 2020; 30: 3042026.

[6] Restarting Cervical Screening Programs during and after the COVID 19 Pandemic. HPV World. Year. Available at: www.hpvworld.com/articles/restarting-cervical-screening-p rograms-during-and-after-the-covid-19-pandemic (Accessed: 10 October 2020).

[7] Steben, M., Norris, T., Rosberger, Z., HPV Global Action. COVID-19 Won't Be the Last (Or Worst) Pandemic: It's Time to Build Resilience Into Our Cervical Cancer Elimination Goals. Journal of Obstetrics and Gynaecology Canada. 2020; 42: 11951196.

[8] Desai KT, Ajenifuja KO, Banjo A, Adepiti CA, Novetsky A, Sebag C, et al. Design and feasibility of a novel program of cervical screening in Nigeria: self-sampled HPV testing paired with visual triage. Infectious Agents and Cancer. 2020; 15: 60.

[9] Ajenifuja KO, Belinson J, Goldstein A, Desai KT, de Sanjose S, Schiffman M. Designing low-cost, accurate cervical screening strategies that take into account COVID-19: a role for selfsampled HPV typing2. Infectious Agents and Cancer. 2020; 15: 61.

[10] Kumar Y, Gupta S, Singla R, Hu YC. A systematic review of artificial intelligence techniques in cancer prediction and diagnosis. Archives of Computational Methods in Engineering. 2021; $1-28$.

[11] Xue P, Tang C, Li Q, Li Y, Shen Y, Zhao Y, et al. Development and validation of an artificial intelligence system for grading colposcopic impressions and guiding biopsies. BMC Medicine. 2020; 18: 406.

[12] Xue P, Ng MTA, Qiao Y. The challenges of colposcopy for cervical cancer screening in LMICs and solutions by artificial intelligence. BMC Medicine. 2020; 18: 169.

[13] Li Y, Chen J, Xue P, Tang C, Chang J, Chu C, et al. ComputerAided Cervical Cancer Diagnosis Using Time-Lapsed Colposcopic Images. IEEE Transactions on Medical Imaging. 2020; 39: 3403-3415.

[14] Li Y, Liu ZH, Xue P, Chen J, Ma K, Qian T, et al. GRAND A large-scale dataset and benchmark for cervical intraepithelial Neoplasia grading with fine-grained lesion description. Medical Image Analysis. 2021; 70: 102006.

[15] Long M, Chantigian PDM, Casey P, Henry M, Schwartz K, Boerger A, et al. 2021 ASCCP Oral Presentations. Journal of Lower Genital Tract Disease. 2021; 25: 1-8.

[16] Ordos, Inner Mongolia promotes free vaccination against cervical cancer-chinanews.com. Teller Report. Year. Available at: https://www.tellerreport.com/life/2021-01-19-\%0A---o rdos--inner-mongolia-promotes-free-vaccination-against-c ervical-cancer-chinanews-com\%0A--.HymqpNONJ_html (Accessed: 19 January 2021). 